CONFORMAL GEOMETRY AND DYNAMICS

An Electronic Journal of the American Mathematical Society

Volume 3, Pages 151-157 (November 30, 1999)

S $1088-4173(99) 00051-\mathrm{X}$

\title{
NONLINEAR AUTOMORPHISMS OF PLANE DOMAINS
}

\author{
TIMO ERKAMA
}

\begin{abstract}
We prove that the number of holomorphic nonlinear polynomials mapping a plane domain one-to-one onto itself is at most countable.
\end{abstract}

\section{INTRODUCTION}

Several symmetric plane domains have a nondiscrete group of automorphisms defined by holomorphic polynomials of degree one. Examples of such domains are the whole complex plane $\mathbf{C}$, a disk, a strip and an annulus. A strip has a continuous group of automorphisms defined by monic polynomials whereas the unit disk $U=\{z \in \mathbf{C}|| z \mid<1\}$ and the upper half plane $H=\{z \in \mathbf{C} \mid \operatorname{Im} z>0\}$ have a one-dimensional group of automorphisms defined by homogeneous polynomials of degree one. A complete characterization of domains with the above property appears in 4 .

Some nonlinear polynomials can also map a planar domain one-to-one onto itself. For example, if a nonlinear polynomial is holomorphically conjugate to a rotation in a neighborhood of a neutral fixed point, then this point is the center of a Siegel disk $D$ and the polynomial generates a nondiscrete semigroup of holomorphic automorphisms of $D$. Another example of automorphisms defined by nonlinear polynomials is studied in Section 5 .

For any subdomain $D$ of $\mathbf{C}$ let $\operatorname{Pol}(D)$ be the set of all holomorphic polynomials mapping $D$ one-to-one onto itself. Then $\operatorname{Pol}(D)$ is a semigroup with a topology induced by the Lie group $\operatorname{Aut}(D)$ of all holomorphic automorphisms of $D$.

Theorem 1. For each $k \geq 2$ the set $\{P \in \operatorname{Pol}(D) \mid \operatorname{deg} P=k\}$ is discrete.

Corollary 1. $\operatorname{Pol}(D)$ contains at most countably many nonlinear polynomials.

Corollary 1 is an immediate consequence of Theorem 1 because Aut $(D)$ is second countable. Hence $\operatorname{Pol}(D)$ can be uncountable only if it has a nondiscrete subgroup of linear polynomials; then $D$ is one of the symmetric domains described in [4].

If $\operatorname{Aut}(D)$ is not discrete, then $D$ is either simply or doubly connected. In both cases each nonlinear element of $\operatorname{Pol}(D)$ is conformally conjugate to an elliptic, parabolic or hyperbolic Möbius transformation. In the elliptic case $D$ is an invariant subdomain of a Siegel disk; the example in Section 5 deals with the hyperbolic case.

The proof of Theorem 1 is based in the study of polymorphisms introduced in 6]. A polymorphism of a nonconstant holomorphic function $f: H \rightarrow \mathbf{C}$ is a pair $(\phi, P)$ of holomorphic polynomials such that $\phi \in \operatorname{Pol}(H)$ and $f \circ \phi=P \circ f$. The

Received by the editors June 23, 1999 and, in revised form, September 24, 1999.

1991 Mathematics Subject Classification. Primary 30D05; Secondary 58F23.

(C)1999 American Mathematical Society 
set $\Pi(f)$ of all polymorphisms of $f$ is a topological semigroup with the topology induced by the map $(\phi, P) \mapsto \phi$ from $\Pi(f)$ to $\operatorname{Pol}(H)$. In [6] we proved

Theorem 2. For each $k \geq 2$ the set $\{(\phi, P) \in \Pi(f) \mid \operatorname{deg} P=k\}$ is discrete unless $f$ is the composition of the exponential function $e^{z}$ and two linear polynomials.

The property of Theorem 2 can be used to characterize the exponential function also in the whole complex plane $\mathbf{C}$ [5] Theorem 2].

We assume that the reader is familiar with complex analytic geometry; this will be needed in the study of some complex analytic subsets of $\operatorname{Aut}(\mathbf{C} \cup\{\infty\})$. For the terminology we refer to 2 .

It is well known that all nonelementary groups of Möbius transformations contain loxodromic elements. We start with a corresponding result for semigroups which we shall need in Section 4 .

\section{Semigroups of $S L(2, \mathbf{R})$}

Let $S L(2, \mathbf{R})$ be the multiplicative Lie group of real $2 \times 2$-matrices with determinant one. An element of $S L(2, \mathbf{R})$ is hyperbolic if it has two real distinct eigenvalues.

Lemma 1. Suppose that a subset $\Gamma \subset S L(2, \mathbf{R})$ is a multiplicative semigroup. Then either $\Gamma$ is commutative or $\Gamma$ contains a hyperbolic element.

Proof. Suppose that $\Gamma$ contains two elements $A_{1}$ and $A_{2}$ which do not commute. We have to prove that $\Gamma$ contains a hyperbolic element.

Recall that an element $A \in S L(2, \mathbf{R})$ is hyperbolic if and only if the trace of $A$ satisfies $|\operatorname{tr} A|>2$. If $A$ is not hyperbolic, then either $A$ or $-A$ is conjugate to

$$
\left(\begin{array}{ll}
1 & b \\
0 & 1
\end{array}\right) \text { or }\left(\begin{array}{rr}
\cos \theta & \sin \theta \\
-\sin \theta & \cos \theta
\end{array}\right) \text {. }
$$

Suppose first that $A_{1}$ has exactly one real eigenvalue and that $A_{2}$ is not hyperbolic. By conjugation (and if necessary replacing $A_{1}$ by $A_{1}^{2}$ ) we may assume that $A_{1}=I+b e_{1} e_{2}^{T}$ where $e_{1}$ and $e_{2}$ are the first and second columns of the identity matrix $I$, respectively. Then for each positive integer $k$ the binomial theorem implies that $A_{1}^{k} A_{2}=A_{2}+k b e_{1} e_{2}{ }^{T} A_{2}$. Moreover, the trace of $b e_{1} e_{2}{ }^{T} A_{2}$ is nonzero, because $A_{1}$ and $A_{2}$ do not commute and $A_{2}$ is not hyperbolic. We conclude that $\left|\operatorname{tr}\left(A_{1}{ }^{k} A_{2}\right)\right|>2$ if $k$ is large enough, so that $A_{1}{ }^{k} A_{2}$ is hyperbolic.

It remains to consider the case when $A_{1}$ and $A_{2}$ have no real eigenvalues. Then $A_{1}$ and $A_{2}$ are both conjugate to a matrix of the form

$$
\left(\begin{array}{rr}
\cos \theta & \sin \theta \\
-\sin \theta & \cos \theta
\end{array}\right)
$$

so that $A_{1}^{-1}$ and $A_{2}^{-1}$ can be approximated by positive powers of $A_{1}$ and $A_{2}$, respectively. More precisely, for $i=1,2$ every neighborhood of $A_{i}{ }^{-1}$ contains a power of $A_{i}$. Since $A_{1}$ and $A_{2}$ do not commute, their commutator $A_{1} A_{2} A_{1}{ }^{-1} A_{2}{ }^{-1}$ is hyperbolic [3, Lemma 3.2] and can be approximated by elements of $\Gamma$ of the form $A_{1} A_{2} A_{1}{ }^{k} A_{2}{ }^{l}$ for suitable positive integers $k$ and $l$. Since the set of hyperbolic elements is open in $S L(2, \mathbf{R})$, we conclude that $A_{1} A_{2} A_{1}{ }^{k} A_{2}{ }^{l}$ is hyperbolic for some $k, l$. This completes the proof of Lemma 1 . 
We finally mention that there is an epimorphism from $S L(2, \mathbf{R})$ to the complex analytic Lie group $\operatorname{Aut}(\mathbf{C} \cup\{\infty\})$ of holomorphic automorphisms of the Riemann sphere. The image of $A=\left(\begin{array}{ll}a & b \\ c & d\end{array}\right)$ under this epimorphism will be denoted by $A_{*} ;$ then

$$
A_{*}(z)=\frac{a z+b}{c z+d} \quad \text { for each } \quad z \in \mathbf{C} \cup\{\infty\} .
$$

\section{Holomorphic FAMilies of FUnCTIONAL EQUATIONS}

For each subdomain $G \subset \mathbf{C}$ let $H(G)$ denote the algebra of holomorphic functions of $G$.

Let $N$ be a complex analytic subset of $\operatorname{Aut}(\mathbf{C} \cup\{\infty\})$, and let $f \in H(U)$ be nonconstant. We say that a map $\beta: N \rightarrow H(f(U))$ is a holomorphic family of functional equations of $f$ if there is a subdomain $V \subset U$ such that $\phi(V) \subset U$ for each $\phi \in N$ and

$$
f(\phi(z))=\beta(\phi)(f(z))
$$

for each $(\phi, z) \in N \times V$.

Theorem 3. Suppose that $\beta: N \rightarrow H(f(U))$ is a holomorphic family of functional equations of a nonconstant holomorphic function $f \in H(U)$, and suppose that $N$ has an accumulation point $\phi_{0} \in N$ such that $\phi_{0}(U)=U$. Then $f$ has a holomorphic extension to all but at most two points of $\partial U$.

The function $f(z)=\log \frac{1+z}{1-z}$ provides an example of a situation where the hypotheses of Theorem 3 are satisfied but $f$ fails to have a holomorphic extension to two points of $\partial U$. In this case $N$ consists of Möbius transformations of the form $\phi_{\alpha}(z)=\frac{z-\alpha}{1-\alpha z}$ where $|\alpha|<\frac{1}{2}$, and $\beta\left(\phi_{\alpha}\right)(w)=w-f(\alpha)$ for each $w \in f(U)$.

Proof. For each $\phi \in N$ we can define a holomorphic extension of $f$ to $U \cup \phi(U)$ such that (1) holds for each $z \in U$. By replacing $N$ with a sufficiently small subspace of $N$ we may assume that for each $\phi \in N$ the closure of $\phi(U)$ contains the origin but does not contain the point at infinity. Then for each $\omega \in \partial U$ there is a holomorphic function $\Phi_{\omega}: N \rightarrow \mathbf{C}$ such that

$$
\Phi_{\omega}(\phi)=\phi(\omega) \quad(\phi \in N) .
$$

Let $V$ be the set of all points $\omega \in \partial U$ such that $\Phi_{\omega}$ is locally constant at $\phi_{0}$. Then $V$ contains at most two points, because elements of $N$ are uniquely determined by their values at any three distinct points.

Let $\omega_{1}, \omega_{2}$ and $\omega_{3}$ be three distinct points of $\partial U$ such that $\omega_{1} \in \phi_{0}(V)$ if $V$ is not empty. It suffices to prove that $f$ has a holomorphic extension to either $\omega_{2}$ or $\omega_{3}$.

If $V$ is empty, choose $\omega \in \partial U$ such that $\phi_{0}(\omega)=\omega_{2}$. Since $\Phi_{\omega}$ is not locally constant at $\phi_{0}$, by the maximum principle [8] p. 234] the image of $\Phi_{\omega}$ contains an open neighborhood of $\phi_{0}(\omega)=\omega_{2}$. Thus there exists $\phi \in N$ and $\omega_{0} \in \partial U$ such that $\left|\phi\left(\omega_{0}\right)\right|>1$ and $\arg \phi\left(\omega_{0}\right)=\arg \omega_{2}$. Since the closure of $\phi(U)$ contains $\phi\left(\omega_{0}\right)$ and the origin, by convexity the point $\omega_{2}$ on the line segment joining $\phi\left(\omega_{0}\right)$ and 0 is contained in $\phi(U)$. Hence $f$ has a holomorphic extension to $\omega_{2}$.

If $V$ is not empty, we choose $h \in \operatorname{Aut}(\mathbf{C} \cup\{\infty\})$ such that $h(U)=H, h(\infty)=-i$ and $h\left(\omega_{1}\right)=\infty$. Let $x$ be any point between $h\left(\omega_{2}\right)$ and $h\left(\omega_{3}\right)$ on the real axis such 
that $h^{-1}(x) \notin \phi_{0}(V)$, and let $\omega=\phi_{0}{ }^{-1}\left(h^{-1}(x)\right)$. Then $\Phi_{\omega}$ is not locally constant at $\phi_{0}$, so that again by the maximum principle $\Phi_{\omega}$ maps every neighborhood of $\phi_{0}$ to a neighborhood of $h^{-1}(x)$. Thus there exists $\phi \in N$ such that $h(\phi(\omega))$ is an interior point of the triangle $T$ with vertices at $h\left(\omega_{2}\right), h\left(\omega_{3}\right)$ and $-i$, and by choosing $\phi$ close to $\phi_{0}$ we may assume that $\phi^{-1}\left(\omega_{1}\right)=\phi_{0}^{-1}\left(\omega_{1}\right)$, because $\omega_{1} \in \phi_{0}(V)$. Then $h(\phi(U))$ is a half plane containing at least one vertex of $T$, because the point $h(\phi(\omega))$ on the boundary of $h(\phi(U))$ is an interior point of $T$. On the other hand, the vertex $-i$ of $T$ is not contained in $h(\phi(U))$, so that either $h\left(\omega_{2}\right)$ or $h\left(\omega_{3}\right)$ is a point of $h(\phi(U))$. Thus either $\omega_{2}$ or $\omega_{3}$ is contained in $\phi(U)$, so that $f$ has a holomorphic extension to $\omega_{2}$ or $\omega_{3}$. This completes the proof of Theorem [3,

\section{Proof of Theorem 1}

Suppose that the set $\{P \in \operatorname{Pol}(D) \mid \operatorname{deg} P=k\}$ is not discrete for some $k$; we have to prove that $k=1$. Since $\operatorname{Pol}(D)$ is a subset of $\operatorname{Aut}(D)$, it follows that $\operatorname{Aut}(D)$ is not discrete. It is well known that $\operatorname{Aut}(D)$ can be nondiscrete only if $D$ is either simply or doubly connected [10. Thus we may assume that there exists a holomorphic isomorphism $f: G \rightarrow D$ where $G$ is either the whole plane, a punctured plane, a disk, a punctured disk, or an annulus.

Let $N_{0}$ be the set of all $\phi \in \operatorname{Aut}(G)$ such that $f \circ \phi \circ f^{-1}$ is the restriction of a polynomial of degree $\leq k$; then $N_{0}$ is not discrete in $\operatorname{Aut}(G)$. Let us first consider the case when the semigroup $\left\langle N_{0}\right\rangle$ generated by $N_{0}$ is commutative.

The number of polynomials of degree $k$ commuting with a given nonlinear polynomial is finite [7. Since $\left\langle N_{0}\right\rangle$ is commutative, it follows that $f \circ \phi \circ f^{-1}$ can be the restriction of a nonlinear polynomial only for finitely many $\phi \in N_{0}$. Since $N_{0}$ is not discrete, we conclude that $k=1$. Thus we may assume that $\left\langle N_{0}\right\rangle$ is not commutative.

If $G$ is the whole complex plane $\mathbf{C}$, the same is true of $D$ and there is nothing to prove. If $G$ is a punctured plane, a punctured disk or an annulus, then $f$ can be chosen so that $f$ linearizes each element of $\operatorname{Pol}(D)$, i.e. $f^{-1} \circ P \circ f$ is the restriction of a linear polynomial for each $P \in \operatorname{Pol}(D)$. In this case the component of the identity of $\operatorname{Aut}(G)$ is abelian and contains $N_{0}$, so that $\left\langle N_{0}\right\rangle$ is commutative. Hence it remains to consider the case when $G$ is a disk, and we may of course assume that $G$ is the open unit disk $U$. Then each element of $N_{0}$ is the restriction of an element of $\operatorname{Aut}(\mathbf{C} \cup\{\infty\})$, and from now on we shall identify $N_{0}$ with the corresponding subset of $\operatorname{Aut}(\mathbf{C} \cup\{\infty\})$.

Let $V=\left\{z \in U|| z \mid<\frac{1}{2}\right\}$ and let $\mathfrak{M}=\{\phi \in \operatorname{Aut}(\mathbf{C} \cup\{\infty\}) \mid \phi(V) \subset U\}$; then $\mathfrak{M}$ is open in $\operatorname{Aut}(\mathbf{C} \cup\{\infty\})$, and the map

$$
(\phi, w) \mapsto\left(f \circ \phi \circ f^{-1}\right)(w)
$$

is holomorphic in $\mathfrak{M} \times f(V)$. The partial derivatives of this map with respect to $w$ are also holomorphic, and for each fixed $w \in f(V)$ we can define a holomorphic function $F_{w}: \mathfrak{M} \rightarrow \mathbf{C}$ such that

$$
F_{w}(\phi)=\frac{\partial^{k+1}}{\partial w^{k+1}}\left(f \circ \phi \circ f^{-1}\right)(w)
$$

for each $\phi \in \mathfrak{M}$. Let

$$
N=\left\{\phi \in \mathfrak{M} \mid F_{w}(\phi)=0 \text { for each } w \in f(V)\right\} ;
$$

then $N$ is a complex analytic subset of $\mathfrak{M}[2]$. 
From the definition of $N$ it follows that for each $\phi \in N$ there is a polynomial $P_{\phi}$ of degree $\leq k$ such that

$$
P_{\phi}(w)=\left(f \circ \phi \circ f^{-1}\right)(w) \text { for each } w \in f(V) .
$$

Then

$$
f \circ \phi(z)=P_{\phi} \circ f(z)
$$

for each $z \in V$. It is also clear that $N_{0} \subset N$, so that $N$ has an accumulation point $\phi_{0} \in N$ such that $\phi_{0}(U)=U$. Moreover, the map $\phi \mapsto P_{\phi}$ is a holomorphic family of functional equations of $f$. From Theorem 3 it follows that $f$ has a holomorphic extension to all but at most two points of $\partial U$.

Let $\Omega$ be the set of points $\omega \in \partial U$ such that $f$ does not have a holomorphic extension to $\omega$. It is clear that $\phi^{-1}(\Omega) \subset \Omega$ for each $\phi \in N_{0}$; in fact, it follows from (2) that if $f$ does not have a holomorphic extension to $\omega$, then $f$ does not have a holomorphic extension to $\phi^{-1}(\omega)$ either. But $\phi^{-1}(\Omega) \subset \Omega$ implies that

$$
\Omega \subset \phi(\Omega),
$$

and since $\Omega$ and $\phi(\Omega)$ have the same cardinality, we conclude that

$$
\Omega=\phi(\Omega)
$$

for each $\phi \in N_{0}$.

Let us first consider the case when $\Omega$ is empty, so that $f$ has a holomorphic extension to a domain $U_{1}$ containing the closure of $U$. Let $\Gamma$ be the set of all $A \in S L(2, \mathbf{R})$ such that $A_{*} \in\left\langle N_{0}\right\rangle$ where $A_{*}$ is defined as in Section 2 Then $\Gamma$ is a multiplicative semigroup. Since $\left\langle N_{0}\right\rangle$ is not commutative, the same is true of $\Gamma$, and by Lemma $1 \Gamma$ contains a hyperbolic element $A$.

Since $A_{*} \in\left\langle N_{0}\right\rangle$, there exist $\phi_{1}, \ldots, \phi_{n} \in N_{0}$ such that $A_{*}=\phi_{1} \circ \cdots \circ \phi_{n}$, and by recalling the definition of $N_{0}$ we see that $f \circ A_{*} \circ f^{-1}$ is the restriction of a polynomial $P$. Therefore

$$
f \circ A_{*}=P \circ f
$$

in $U$. By analytic continuation this equation defines a holomorphic extension of $f$ to $A_{*}\left(U_{1}\right),\left(A^{2}\right)_{*}\left(U_{1}\right)$, and by induction, to $\left(A^{n}\right)_{*}\left(U_{1}\right)$ for each positive integer $n$. But since $A$ is hyperbolic, these sets cover the whole Riemann sphere. However, this is not possible because all holomorphic functions of $\mathbf{C} \cup\{\infty\}$ are constant. We conclude that $\Omega$ is not empty.

Since $\Omega$ contains at most two points, it follows from (3) that $\phi(\phi(\omega))=\omega$ for each $\phi \in N_{0}$ and each $\omega \in \Omega$. Choose $h \in \operatorname{Aut}(\mathbf{C} \cup\{\infty\})$ such that $h(U)=H$ and $h^{-1}(\infty) \in \Omega$. Then $h \circ \phi \circ \phi \circ h^{-1} \in \operatorname{Pol}(H)$ for each $\phi \in N_{0}$, and (2) implies that the set

$$
\left\{\left(h \circ \phi \circ \phi \circ h^{-1}, P_{\phi} \circ P_{\phi}\right) \mid \phi \in N_{0}\right\}
$$

is a subset of $\Pi\left(f \circ h^{-1}\right)$. Moreover, $h \circ \phi \circ \phi \circ h^{-1}$ can be the identity of $\operatorname{Pol}(H)$ only if $\operatorname{deg} P_{\phi}=1$. It follows that either $k=1$ or $\left(h \circ \phi_{0} \circ \phi_{0} \circ h^{-1}, P_{\phi_{0}} \circ P_{\phi_{0}}\right)$ is an accumulation point of (44). Also, $f \circ h^{-1}$ cannot be the composite of $e^{z}$ and two linear polynomials, because $f \circ h^{-1}$ is univalent in $H$. In view of Theorem 2 we conclude that $k=1$. The proof of Theorem 1 is now complete. 


\section{An eXAmple}

In this section we construct a plane domain where the quadratic polynomial

$$
P(z)=z^{2}+\frac{1}{2} z
$$

is holomorphically conjugate to a hyperbolic Möbius transformation. The example shows that a discrete group generated by a holomorphic polynomial need not be a Kleinian group.

Let $g$ be an entire function linearizing $P$ at the repelling fixed point $z=\frac{1}{2}$. Then $g(0)=\frac{1}{2}$,

$$
g\left(\frac{3}{2} z\right)=P(g(z))
$$

for each $z \in \mathbf{C}$, and we may assume that $g^{\prime}(0)=-1$. Such linearizing maps have been studied in detail by Myrberg [9].

Let $T$ be the closed triangle with vertices at $\frac{1}{2}$ and $-\frac{1}{16}(1 \pm i)$. A straightforward computation shows that $T$ is forward invariant under $P$, i.e. $P$ maps every point of $T$ into a point of $T$. A study of the branches of $P^{-1}$ shows also that $P$ is one-to-one in $T$.

Since $g$ is conformal at the origin and $g^{\prime}(0)=-1$, there is $\delta>0$ such that $g\left(r e^{i \theta}\right) \in T$ if $0<r<\delta$ and $|\theta|<\delta$. Since $T$ is forward invariant, iteration of (5) shows that $g$ maps the domain

$$
\{\zeta \in \mathbf{C}|| \arg \zeta \mid<\delta\}
$$

onto a subdomain $D$ of $T$. Moreover, $P$ maps $D$ onto itself, so that $P \in \operatorname{Pol}(D)$.

Figure 1 indicates the shape of $D$ as well as some of the orbits and streamlines invariant under $P$. Note that $P$ generates a discrete group of automorphisms of $D$. A result of Azarina 1 implies that for examples of this kind the boundary of $D$ cannot be an analytic curve; accordingly the boundary in Figure 1 is not smooth at the fixed points of $P$.

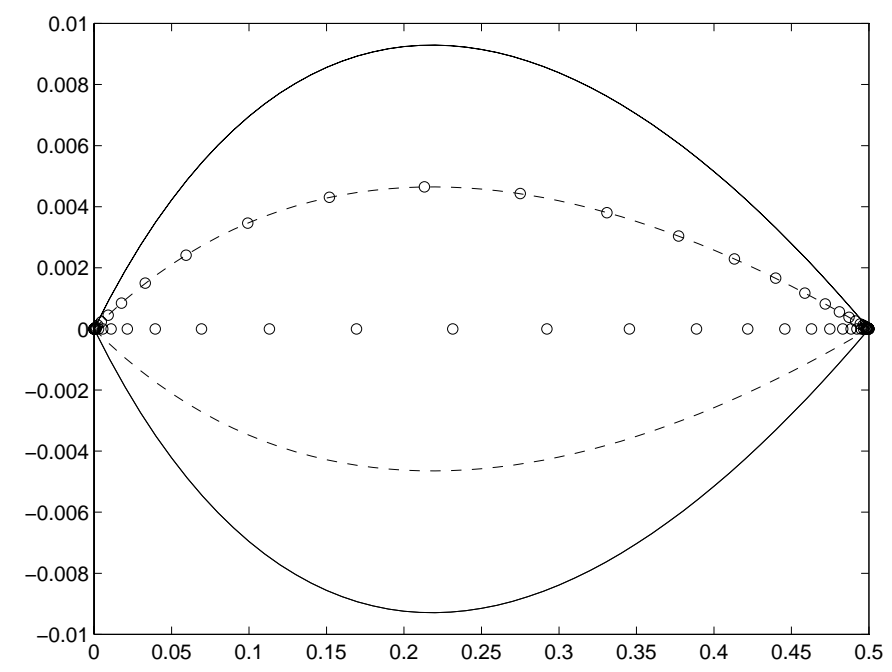

FIGURE 1. 
For this example it is not hard to prove that $\operatorname{Pol}(D)$ is commutative. It is an open question whether for some other domain $\operatorname{Pol}(D)$ could contain two nonlinear elements which do not commute.

\section{REFERENCES}

1. Yu. V. Azarina, Invariant analytic curves for entire functions, Siberian Math. J. 30 (1989), no. 3, 349-353. MR 90j:30038

2. E. M. Chirka, Complex Analytic Sets, Kluwer, 1989. MR 92b:32016

3. T. Erkama, Group actions and extension problems for maps of balls, Ann. Acad. Sci. Fenn. Ser. A I Math. 556 (1973), 1-31. MR 57:624

4. - Möbius automorphisms of plane domains, Ann. Acad. Sci. Fenn. Ser. A I Math. 10 (1985), 155-162. MR 87c:30063

5. - The exponential function and linearization of quadratic polynomials, Analysis, Algebra and Computers in Mathematical Research, Lecture Notes in Pure and Applied Mathematics, Marcel Dekker, 1994, pp. 71-80. MR 95g:39028

6. $ـ$ Polymorphisms and linearization of nonlinear polynomials, Ann. Acad. Sci. Fenn. Ser. A I Math. 22 (1997), 113-121. MR 97j:30008

7. E. Jacobsthal, Über vertauschbare Polynome, Math. Z. 63 (1955), 243-276. MR 17:574a

8. S. Łojasiewicz, Introduction to Complex Analytic Geometry, Birkhäuser, 1991. MR 92g:32002

9. P. J. Myrberg, Über ganze Funktionen mit rationalem Multiplikationstheorem, Ann. Acad. Sci. Fenn. Ser. A I Math. 414 (1968), 1-20. MR 37:4256

10. R. Nevanlinna, Uniformisierung, Springer, 1953. MR 15:208h

University of JoensuU, SF-80101 JoensuU, Finland

E-mail address: Timo.Erkama@joensuu.fi 